Topic Introduction

\title{
Immunoprecipitation
}

\author{
James DeCaprio and Thomas O. Kohl
}

Immunoprecipitation, commonly referred to as IP, involves the binding of proteinaceous antigen in solution by an antigen-specific antibody followed by purification of the antigen-antibody complex via attachment to a solid-phase matrix such as Protein A or G agarose. This rather simplistic and rapid technique yields highly purified immune complexes from multifactorial solutions, including cell lysates or homogenized tissues, and is most commonly used to identify and determine the relative abundance of interacting proteins, referred to as coimmunoprecipitation or co-IP. Although methods encompassing immunoblotting or western blotting of cell lysate preparations can also be applied to determine the presence and quantity of a specific antigen, its relative molecular weight, rate of synthesis or degradation, and state of target-specific posttranslational modification, immunoprecipitation can significantly increase the sensitivity for these methodologies.

Immunoprecipitation was first described in the 1960s (Barrett et al. 1960). The immunoprecipitation technique shown in Figure 1 is characterized by the following phases: (1) the preparatory phase, including culture of target cells and mechanical or chemical cell lysis to release the antigen of interest (Fig. 1A); (2) the antibody-antigen complex formation phase and purification phase (Fig. 1B); and (3) the immune complex detection phase (Fig. 1C).

Native, transformed, or transfected cells of mammalian or yeast origin as well as animal-derived tissues are most commonly sourced and/or prepared for the release of proteins used in immunoprecipitations. Cells for immunoprecipitation must be cultured and harvested under optimal growth conditions to ensure increased viability while simultaneously preventing nonspecific degradation of cellular proteins. During the culturing process, cells can be enriched specifically for a particular cell cycle phase as well as states of differentiation in response to growth factors, drugs, toxins, DNAdamaging reagents, and other stimuli. Metabolic labeling of antigens can be achieved by supplementing the cell culture medium with radioactive precursors such as $\left[{ }^{35} \mathrm{~S}\right]$ methionine or $\left[{ }^{32} \mathrm{P}\right]$ orthophosphate during the incubation period of target cells (see Protocol: Metabolic Labeling of Protein Antigens with $\left[{ }^{35}\right.$ S]Methionine [DeCaprio and Kohl 2017a] and Protocol: Metabolic Labeling of Protein Antigens with $\left[{ }^{32} \mathrm{P}\right]$ Orthophosphate [DeCaprio and Kohl 2018a], respectively). If cells will be used for chromatin immunoprecipitation (CHIP), an additional fixation step before cell lysis to ensure stabilization of protein-DNA interactions is required (Protocol: Chromatin Immunoprecipitation [DeCaprio and Kohl 2020a]). If needed, cells can be frozen before immunoprecipitation (see Protocol: Freezing Cell Pellets for Large-Scale Immunoprecipitation [Litovchick 2019]).

After cultured cells are harvested, antigens of interest are extracted from cells in lysis buffer to solubilize all of the proteins. Numerous lysis buffer formulations have been developed and specifically serve the purpose of extracting proteins from the cytoplasm, the membrane, and the organelles,

From the Antibodies collection, edited by Edward A. Greenfield.

(C) 2020 Cold Spring Harbor Laboratory Press

Cite this introduction as Cold Spring Harb Protoc; doi:10.1101/pdb.top098509 
J. DeCaprio and T.O. Kohl
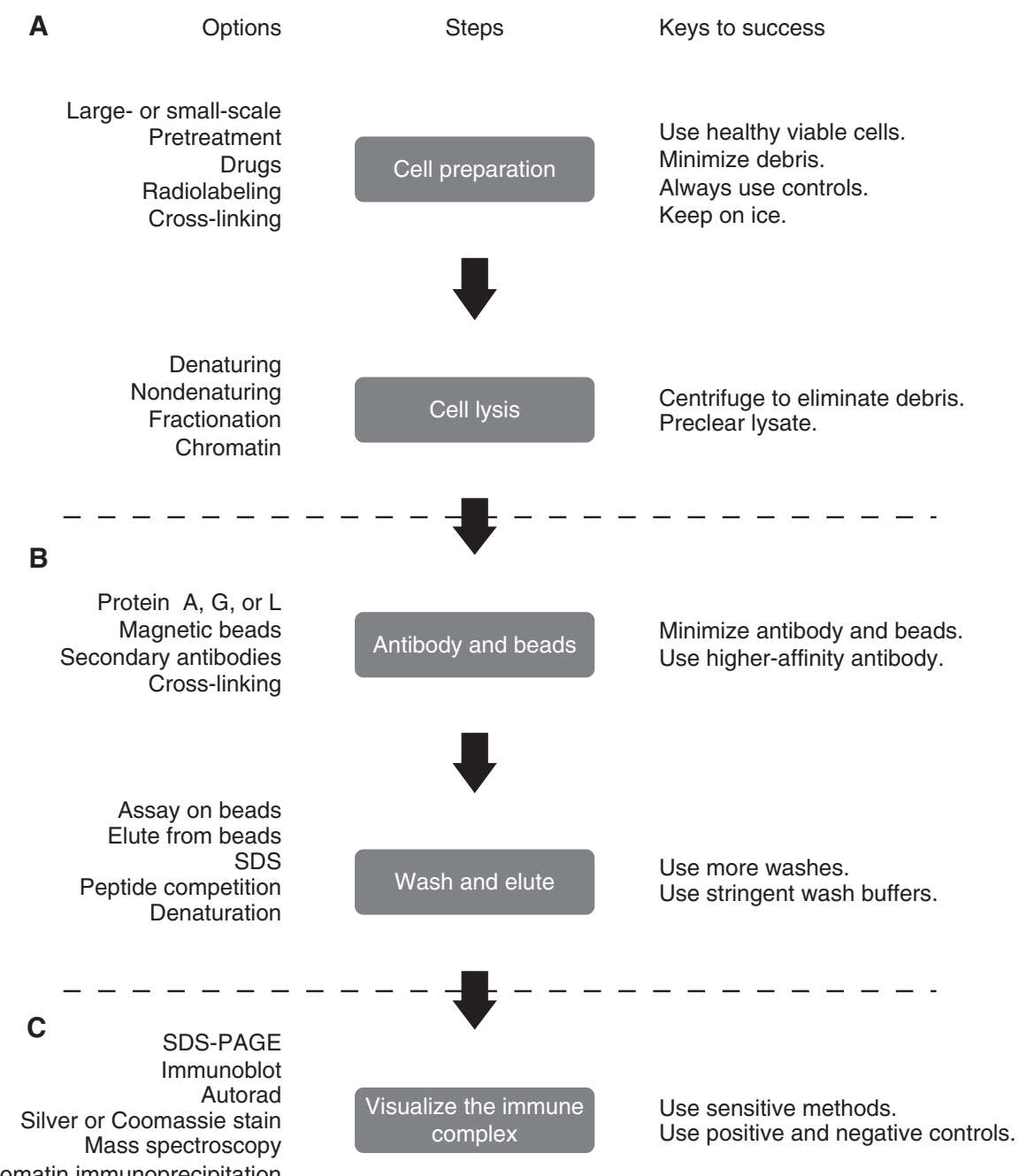

FIGURE 1. Immunoprecipitation experimental strategies. (A) The preparatory phase, $(B)$ the antibody-antigen complex, and $(C)$ the immune complex detection phase. (SDS) Sodium dodecyl sulfate; (SDS-PAGE) sodium dodecyl sulfate polyacrylamide gel electrophoresis.

including the nucleus, or chromatin (see Protocol: Differential Detergent Lysis of Cellular Fractions for Immunoprecipitation [DeCaprio and Kohl 2020b]). The effect of lysis buffers on the cellular environment must be matched to the antibody used for capturing the antigen because particular antibodies are only capable of binding denatured epitopes (see Protocol: Denaturing Lysis of Cells for Immunoprecipitation [DeCaprio and Kohl 2020c]), whereas others are conformation specific and will only bind to proteins in their native form. Lysis buffers applied for investigations of proteinspecific posttranslational modifications (PTMs) differ from those described above and contain specific inhibitors that can preserve the modified state of an immunoprecipitated protein antigen and associated proteins. During and after the cell lysis process, phosphatases and proteases remain active and may contribute to degradation of the protein of interest; thus, contamination of lysates with particulate matter and cellular debris must be avoided to increase the quality of an immunoprecipitation. Removal of nonspecific proteins from the cell lysate greatly increases target-specific detection in the final analysis.

After the cell lysate has been produced, antigen-specific antibodies are added to allow formation of the antibody-antigen complex. A solid-phase matrix such as Protein A or G agarose is subsequently added, and the formed immune complexes are allowed to bind by adsorption, facilitated by an 
interaction of the antibody with the agarose bead resin. Once the antigen-antibody-bead resin interaction has occurred, unbound nonspecific proteins are removed through a wash step of the resin, leaving behind the purified antibody-antigen complexes bound to the solid-phase matrix. Repeated washing steps of the resin greatly reduce the amount of nonspecific protein not directly bound to either antibody or protein antigen and result in highly purified protein antigen-antibody complexes from which the protein antigen can be eluted in various ways. Separation of resin-bound antibody and antigen in the elution step can be achieved in numerous ways-for example, by denaturation in the presence of $2 \%$ sodium dodecyl sulfate (SDS) or by competitive nature in which an excess of peptide, specific to the antibody, is added to the Elution buffer and competes for antibody-binding sites, thus releasing the protein antigen. The antibody bound to the solid-phase matrix is left behind; however, the matrix can be voided of the bound antibody and used repeatedly according to the resin manufacturer's recommendation.

Upon elution, immunoprecipitated proteins in either concentrated or unconcentrated form are most commonly analyzed by one-dimensional (1D) SDS-polyacrylamide gel electrophoresis (SDSPAGE) and visualized by immunoblotting (see Introduction: Immunoblotting [Litovchick 2020]); additional identification by mass spectrometry is an alternative. Furthermore, immunoprecipitated proteins are often incorporated into various other techniques, including enzymatic studies, ligand binding, or other immunochemical methods, thereby greatly enhancing the information obtained from target antigens initially prepared by this simplistic procedure.

The quality of the cellular preparation is the most important factor contributing to the success of an immunoprecipitation, where the immunoprecipitation's sensitivity is governed by the target protein concentration and the specificity is dependent on the amount of cellular debris observed in the cell lysate. The concentration of a cellular target may depend on the proliferation state or cell cycle at the time of lysis; however, cell culture optimization may lead to increased target expression levels. This, in turn, may result in the use of lesser amounts of cell lysate during the analysis, thereby reducing the nonspecific signal. Stable or transfected cell lines and tissues known to express higher levels of specific target protein may represent a better choice for the preparation of a cell lysate for immunoprecipitation purposes.

A "clean" immunoprecipitation shows a strong target signal with little or no background signal, whereas in a "dirty" immunoprecipitation, the target signal cannot be differentiated from the background. This is highly dependent on the insoluble material present in cell lysates consisting of chromatin, cellular membrane fragments, or fibrous material left behind from tissue preparations, which contribute to an increase of nonspecific proteins in the immunoprecipitation and will significantly degrade the quality of an immunoprecipitation in the final analysis. Particular attention should also be paid to the antibody preparation in use, for it may possibly contain debris or microorganisms if prepared and stored without addition of preservatives. A simple centrifugation step can remove debris from the antibody preparation if warranted.

Radiolabeling of protein antigens can be achieved by addition of radioactive amino acids or amino acid precursors to the medium formulation of actively growing cells. Although techniques such as immunoblotting (see Introduction: Immunoblotting [Litovchick 2020]) have largely replaced radiolabeling for the detection of immunoprecipitated proteins, several uses of this technique have not been challenged. Pulse-chase labeling of antigens with $\left[{ }^{35} \mathrm{~S}\right]$ methionine is used to determine the relative half-life of a protein (see Protocol: Pulse-Chase Labeling of Protein Antigens with $\left[{ }^{35} \mathrm{~S}\right]$ Methionine [DeCaprio and Kohl 2018b]), whereas metabolic labeling incorporating $\left[{ }^{32} \mathrm{P}\right]$ orthophosphate has been described as a sensitive method for determining the antigen's state of phosphorylation (see Protocol: Metabolic Labeling of Protein Antigens with $\left[{ }^{32} \mathrm{P}\right.$ ]Orthophosphate [DeCaprio and Kohl 2018a]). Radiolabeling can be combined with immunoblotting, in which an immunoprecipitated radiolabeled target protein is transferred onto a nitrocellulose or polyvinylidene fluoride (PVDF) 
membrane followed by western blotting using an antibody to the same protein. This approach helps to confirm that a specifically radiolabeled protein is truly the target protein.

\section{CELL LYSIS}

Several different techniques can be used to break open cells for the preparation of cell lysates. Treatment options for cells without cell walls include the use of detergents or mechanical force, such as a dounce homogenizer, to disrupt plasma membranes for release of the cellular contents. If cells contain a cell wall (plant, yeast, or bacterium-derived), this must first be digested enzymatically or sheared mechanically before the addition of detergents to release the target antigens contained in the cellular contents. Alternatively, the option of treating whole cells with strong denaturing solutions may be used.

Several different lysis buffers can be used to release protein antigens from cells; however, no single buffer is sufficient for all purposes. Two important considerations should be adhered to when choosing a lysis buffer: (1) efficient release of the antigen of interest and (2) retaining the capability of the antigen to be recognized and bound by the antibody.

When a new antigen is investigated, several different lysis buffers should be evaluated for the extraction of the antigen to determine the optimal buffer composition for efficient antigen extraction with minimal degradation. A good strategy is first to lyse cells using a relatively strong buffer such as radioimmunoprecipitation assay buffer (commonly referred to as RIPA buffer) containing the ionic detergents SDS and sodium deoxycholate (DOC). If successful, these results can be compared with the preparation of a cell lysate under milder conditions containing nonionic detergents such as Nonidet P-40 or Triton X-100. Quantitative release can be judged by comparing antigen detected in the soluble lysate supernatant with that found in the remaining cell pellet after centrifugation and can easily be performed by immunoblotting (see Introduction: Immunoblotting [Litovchick 2020]).

The choice of lysis buffer can greatly influence the outcome of the immunoprecipitation. When priorities have been set to preserve protein-protein interactions or specific enzyme activity, this, in turn, necessitates cellular lysis under milder conditions to preserve the antigen structure and associated protein-protein interactions without causing denaturation. Several key factors relating to the antigen of choice represent decision points in the choice of lysis buffer: (1) antigen location within or on the cell surface, bound to either the membrane or chromatin; (2) antigen localization within the cell (single or multiple locations); and (3) the capability of the buffer to extract all or only part of the antigen. A variety of different lysis buffers can be chosen to selectively extract antigens from distinct cellular locations and organelles while simultaneously reducing the amount of nonspecific interaction with the antigen or differentially affecting the antigen and nonspecific proteins. Furthermore, the choice of lysis buffer may affect the possibility that the extracted antigen might undergo modifications after lysate preparation; these include the possible degradation by proteases or modifications brought forth by kinases, phosphatases, and other enzymes present in the lysate. Most important, however, are the effects conferred by the choice of lysis buffer on the antigen itself that could possibly induce conformational alterations.

In general, conditions used for lysing cells or tissues should be as gentle as possible to retain target antibody-binding sites and to avoid solubilization of cytoskeletal proteins, but harsh enough to ensure quantitative release of the antigen. This translates into choosing nonionic detergents such as Nonidet $\mathrm{P}-40$ or Triton X-100 ranging in concentration from $0.1 \%$ and $2 \%(\mathrm{v} / \mathrm{v})$ over ionic detergents such as SDS or DOC with concentrations ranging from $0.01 \%$ and $2 \%(\mathrm{v} / \mathrm{v})$. Incorporation of lower detergent concentrations over higher ones as well as single detergents over a mixture of detergents into the lysis buffer is generally preferred. Furthermore, additional variables that could significantly affect the release of proteinaceous antigens include $\mathrm{pH}$, salt concentration, and the presence of divalent cations. Recommendations for lysis buffer composition include salt concentrations ranging up to $1 \mathrm{M}$, divalent cation concentrations ranging up to $10 \mathrm{~mm}$, EDTA concentrations ranging up to $5 \mathrm{~mm}$, and a pH value between 6 and 9. All individual components should be evaluated to achieve optimal conditions for antigen extraction. 
During cell lysis and release of cellular content into the lysis buffer, cellular proteases remain active and could potentially cause severe protein degradation. To lessen the effects on an immunoprecipitation, two approaches should be adhered to: (1) care should always be taken to keep all samples, buffers, and consumables ice-cold by placing them on ice, thereby reducing temperature-dependent protease degradation; and (2) the lysis buffer should be supplemented with commercially available protease inhibitors as listed in Table 1 or cocktails thereof. The two most commonly inhibitors used in immunoprecipitations are aprotinin and phenylmethylsulfonyl fluoride (PMSF); however, a mixture of several different protease inhibitors referred to as a cocktail is better, particularly as a starting point for further tests.

\section{Lysis of Tissue Culture Cells}

The most efficient and preferred method of cell lysis for tissue culture-derived cells is treatment with detergents (Protocol: Detergent Lysis of Tissue Culture Cells for Immunoprecipitation [DeCaprio and Kohl 2017b]). Alternatively, cell membranes can also be broken by physical shearing using potters, blenders, ultrasound generators, or dounce homogenizers; these methods are particularly useful in the processing of cell pellets derived from large volumes or when detergents are to be avoided. Of these, dounce homogenization with glass pestle and mortar (Protocol: Using Dounce Homogenization to Lyse Cells for Immunoprecipitation [DeCaprio and Kohl 2019a]) is by far the gentlest method; however, it is only efficient when homogenizing larger volumes of several milliliters or more. Lysis of cell culture-derived cells by the freeze-thaw method is the least desirable because the repetitive cycle results in excessive proteolytic degradation and denaturation, possibly because of local changes in $\mathrm{pH}$ and osmolarity at the solid-liquid interphase. Unless the antigen of interest is partic-

TABLE 1. Protease inhibitors

\begin{tabular}{|c|c|c|c|c|c|}
\hline Inhibitor & $\begin{array}{l}\text { Protease } \\
\text { target }\end{array}$ & $\begin{array}{l}\mathrm{MW} \\
(\mathrm{Da})\end{array}$ & $\begin{array}{l}\text { Stock solution } \\
\text { (solvent) }\end{array}$ & $\begin{array}{l}\text { Working } \\
\text { concentration (dilution) }\end{array}$ & Comments \\
\hline AEBSF & Serine & 239.5 & $\begin{array}{l}100 \mathrm{mg} / \mathrm{mL}\left(\mathrm{H}_{2} \mathrm{O}\right) \\
400 \mathrm{~mm}\end{array}$ & $\begin{array}{l}0.1-1.0 \mathrm{mg} / \mathrm{mL} \\
0.4-4 \mathrm{~mm}(1000 \times-100 \times)\end{array}$ & Prepare before use \\
\hline Antipain & $\begin{array}{l}\text { Serine } \\
\text { Cysteine }\end{array}$ & 677.6 & $\begin{array}{l}1 \mathrm{mg} / \mathrm{mL}\left(\mathrm{H}_{2} \mathrm{O}\right) \\
1.5 \mathrm{~mm}\end{array}$ & $\begin{array}{l}50 \mu \mathrm{g} / \mathrm{mL} \\
74 \mu \mathrm{M}(20 \times)\end{array}$ & \\
\hline Aprotinin & Serine & 6512 & $\begin{array}{l}10 \mathrm{mg} / \mathrm{mL}\left(\mathrm{H}_{2} \mathrm{O}\right) \\
1.5 \mathrm{mM}\end{array}$ & $\begin{array}{l}1.0 \mu \mathrm{g} / \mathrm{mL} \\
0.15 \mu \mathrm{M}(10,000 \times)\end{array}$ & \\
\hline Benzamidine- $\mathrm{HCl}$ & Serine & 156.6 & $\begin{array}{l}10 \mathrm{mg} / \mathrm{mL}\left(\mathrm{H}_{2} \mathrm{O}\right) \\
64 \mathrm{mM}\end{array}$ & $\begin{array}{l}0.1 \mathrm{mg} / \mathrm{mL} \\
0.6 \mathrm{~mm}(100 \times)\end{array}$ & \\
\hline Bestatin & Amino & 344.8 & $\begin{array}{l}5 \mathrm{mg} / \mathrm{mL} \text { (methanol) } \\
14.5 \mathrm{~mm}\end{array}$ & $\begin{array}{l}40 \mu \mathrm{g} / \mathrm{mL} \\
120 \mu \mathrm{M}(125 \times)\end{array}$ & \\
\hline Chymostatin & Chymotrypsin & 607.7 & $\begin{array}{l}20 \mathrm{mg} / \mathrm{mL} \text { (DMSO) } \\
33 \mathrm{~mm}\end{array}$ & $\begin{array}{l}6-60 \mu \mathrm{g} / \mathrm{mL} \\
10-100 \mu \mathrm{M} \\
(3333 \times-333 \times)\end{array}$ & \\
\hline E64 & Cysteine & 357.4 & $\begin{array}{l}20 \mathrm{mg} / \mathrm{mL}(50 \% \\
\left.\text { ethanol in } \mathrm{H}_{2} \mathrm{O}\right) \\
56 \mathrm{~mm}\end{array}$ & $\begin{array}{l}0.5-10 \mu \mathrm{g} / \mathrm{mL} \\
1-30 \mu \mathrm{M}(40,000 \times \\
-2000 \times)\end{array}$ & \\
\hline EDTA & Metallo & 292.2 & $0.5 \mathrm{M}$ & $\begin{array}{l}0.2-0.5 \mathrm{mg} / \mathrm{mL} \\
0.5-1.3 \mathrm{~mm}(1000 \times)\end{array}$ & $\begin{array}{l}\text { May inhibit enzyme } \\
\text { activity or cause } \\
\text { protein unfolding }\end{array}$ \\
\hline Leupeptin & $\begin{array}{l}\text { Serine } \\
\text { Cysteine }\end{array}$ & 475.6 & $\begin{array}{l}1 \mathrm{mg} / \mathrm{mL}\left(\mathrm{H}_{2} \mathrm{O}\right) \\
2 \mathrm{mM}\end{array}$ & $\begin{array}{l}0.5-2 \mu \mathrm{g} / \mathrm{mL} \\
10-100 \mu \mathrm{M}(2000-500 \times)\end{array}$ & \\
\hline Pepstatin & Acid serine & 685.9 & $\begin{array}{l}1 \mathrm{mg} / \mathrm{mL} \text { (methanol) } \\
1.5 \mathrm{~mm}\end{array}$ & $\begin{array}{l}0.7 \mu \mathrm{g} / \mathrm{mL} \\
1 \mu \mathrm{M}(1500 \times)\end{array}$ & $\begin{array}{l}\text { Useful for animal } \\
\text { tissues and low pH }\end{array}$ \\
\hline PMSF & Serine & 174.2 & $\begin{array}{l}10 \mathrm{mg} / \mathrm{mL} \\
\quad \text { (isopropanol) } \\
60 \mathrm{~mm}\end{array}$ & $\begin{array}{l}17-170 \mu \mathrm{g} / \mathrm{mL} \\
0.1-1 \mathrm{~mm}(600 \times-60 \times)\end{array}$ & $\begin{array}{l}\text { Unstable in } \mathrm{H}_{2} \mathrm{O} \\
t_{1 / 2}=35 \text { min at } \mathrm{pH} 8 \\
\text { Add fresh at every } \\
\text { step. }\end{array}$ \\
\hline 1,10-Phenanthroline & Metallo & 198.2 & $\begin{array}{l}100 \mathrm{mg} / \mathrm{mL} \text { (DMSO) } \\
0.5 \mathrm{M}\end{array}$ & $1-10 \mathrm{~mm}(500 \times-50 \times)$ & \\
\hline
\end{tabular}


J. DeCaprio and T.O. Kohl

ularly resistant to proteolytic cleavage or the efficiency of this method has been validated, it should not be used as a first choice. In general, detergent lysis is the best method for immunoprecipitations.

Lysis of Tissue

Immunoprecipitations of tissues derived from animals can reveal the status of the protein target in a variety of organs and are generally divided into two types - clean and dirty (Protocol: Detergent Lysis of Animal Tissues for Immunoprecipitation [DeCaprio and Kohl 2017c]). Clean immunoprecipitations typically consist of proteins derived from a single cell type such as the eye, embryonic brain, and whole embryo; and dirty immunoprecipitations commonly refer to protein antigens extracted from mammary tissue, adult brain, liver, or carcinomas. The ability to detect a tissue-derived immunoprecipitated protein is highly dependent on its level of expression within the tissue of origin, where higher levels of tissue-specific protein expression will likely yield greater insight and information about the antigen of interest than samples prepared from tissues showing little or no protein expression.

Lysis of Yeast

The preferred method of lysis for yeast cells, including the fission yeast Schizosaccharomyces pombe and the budding yeast Saccharomyces cerevisiae, is by mechanical shearing, either vortexing the cells in the presence of glass beads or by sonication (Protocol: Lysing Yeast Cells with Glass Beads for Immunoprecipitation [DeCaprio and Kohl 2020d] and Protocol: Lysing Yeast Cells for Immunoprecipitation Using a Coffee Grinder [DeCaprio and Kohl 2020e]). This ensures shattering of cell walls, thus bursting the cells and releasing the antigens of interest. Alternatively, enzymatic degradation of cell walls leaves behind spheroplasts that can be lysed with detergent; however, caution is advised with this approach because it might lead to extensive proteolysis. Generally, all lysate preparations should be performed on ice in the presence of protease inhibitors to minimize the effect of proteolytic activity.

Both polyclonal and monoclonal antibodies can be used for immunoprecipitations, and their relative advantages and disadvantages are discussed in detail below. Generally, an antibody is added to the cell lysate before addition of the immunosorbant matrix; this allows for rapid mixing of the liquid-phase cell lysate and antibody solution before addition of the solid-phase matrix. Alternatively, antibody and matrix could be added simultaneously to the cell lysate in separate or preincubated/cross-linked form (see Protocol: Cross-Linking Antibodies to Beads Using Dimethyl Pimelimidate (DMP) [DeCaprio and Kohl 2019b] and Protocol: Cross-Linking Antibodies to Beads with Disuccinimidyl Suberate (DSS) [DeCaprio and Kohl 2019c]).

Immunoprecipitations Using Polyclonal Antibodies

Polyclonal antibody preparations are commonly used for immunoprecipitations and contain a mixture of antibodies capable of binding multiple sites or epitopes on the antigen, therefore displaying a high avidity for the antigen. This is particularly true when a large-molecular-weight protein is used as the antigen in the polyclonal antibody development process, but can even hold true for a relatively short antigenic peptide spanning eight to 20 residues in length. The advantage conferred by the polyclonal antibodies is reflected in the availability of multiple binding sites. This translates not only into the capture of immune complexes, but also into the number of reversible interactions between the polyclonal antibody and the solid-phase matrix, such as Protein A agarose. An increased number of available binding sites for the Protein A molecules facilitates the formation of a more stable antigen-antibody-matrix complex, in which the multiple antibody-antigen and antibody-matrix interactions will generate a stable, multivalent complex that is easily prepared and can be treated relatively harshly during the washing procedure. 
Although the incorporation of polyclonal antibodies in immunoprecipitations often produces stable, multivalent interactions, their use also results in higher nonspecific background in comparison with the use of monoclonal antibodies. Multiple interactions leading to the formation of large complexes are more apt to trap or bind nonspecific proteins.

Polyclonal antibodies, if not purified, are normally used as whole-serum preparations and contain an entire repertoire of circulating antibodies present in the immunized animal at the time of serum collection. Therefore, the serum may contain antibodies that specifically recognize spurious antigens, a particularly troublesome side effect in the analysis of microbial antigens. Sera may contain antibodies raised against bacterial or fungal infections of the host, and if applied for studies involving an organism of identical or similar nature, the subsequent antigen-antibody interactions will contaminate the immunoprecipitation. Similarly, monoclonal antibody-producing hybridomas produced as ascites will contain serum immunoglobulins from the host mouse that could also be reactive against a recent microbial infection, thus highlighting the importance of using healthy, uninfected animals for immunization and antibody development regimen.

On a comparative scale, immunoprecipitations incorporating polyclonal antibodies will normally yield a higher nonspecific background than those incorporating monoclonal antibodies because of contaminating activities and increased nonspecific interactions. This problem is inherent to this technique; however, some of the interfering background can be effectively removed by decreasing the amount of antiserum used to immunoprecipitate the antigen. The nonspecific background can be kept to a minimum by using the smallest amount of serum necessary for the quantitative recovery of the antigen. In addition, stability of the antigen-antibody complexes allows for more stringent washing and may lessen nonspecific background problems. Alternatively, the polyclonal antibody serum can be purified by immunoaffinity using an affinity matrix to enrich for antibodies that are specific to the antigen only.

\section{Immunoprecipitations Using Monoclonal Antibodies}

The greatest advantage of using monoclonal antibodies for immunoprecipitation is that they form highly specific antibody-antigen interactions. Because monoclonal antibodies only bind to one epitope, their use in the identification of a particular structure on an antigen is unsurpassed; they are capable of detecting and differentiating between different forms of the antigen, including conformational changes or posttranslational modifications. Antigen-antibody immune complexes formed by monoclonal antibodies are not usually multimeric and are smaller than those formed by polyclonal antibody-antigen interactions, thus significantly reducing the background typically associated with nonspecific binding.

Monoclonal antibodies used for immunoprecipitations may resolve or lessen the discussed background issues; however, they need to display a high affinity for the antigen if used in an immunoprecipitation. Monoclonal antibodies only facilitate one antibody-antigen interaction, with the exception of multimeric antigens, rendering the affinity of the antibody for its antigen as critically important. Antibodies displaying affinities of $<10^{7} \mathrm{~mol}^{-1}$ are difficult to use in immunoprecipitations, and given that hybridoma-derived antibody panels often include antibodies with affinities as low as $10^{6} \mathrm{~mol}^{-1}$, not all monoclonal antibodies work well in immunoprecipitations. Notably, information regarding the affinity of a monoclonal antibody for a specific target is rarely available.

\section{FORMING AN ANTIBODY-ANTIGEN COMPLEX}

The formation of an antibody-antigen complex is the simplest step in an immunoprecipitation (Protocol: Cross-Linking Antibodies to Beads Using Dimethyl Pimelimidate (DMP) [DeCaprio and Kohl 2019b] and Protocol: Cross-Linking Antibodies to Beads with Disuccinimidyl Suberate (DSS) [DeCaprio and Kohl 2019c]), and the following variables should be considered: (1) amount of antibody to be added, (2) the final volume, and (3) whether or not an anti-immunoglobulin antibody 
will be used. The amount of added antibody is dependent on how much antigen will be precipitated and can be determined by titration of the antibody against a constant amount of antigen. Titration of the polyclonal antibody will help to identify the smallest amount required for the precipitation of the antigen and will often help to reduce the nonspecific background. Although not often required for the use of monoclonal antibodies, recommended starting points for antibody titrations are $0.1-3 \mu \mathrm{L}$ of polyclonal serum, 10-100 $\mu \mathrm{L}$ of hybridoma tissue culture supernatant, or $0.1-1 \mu \mathrm{L}$ of ascites fluid. At the midpoint of these suggested ranges, the amount of specific immunoglobulin will be $\sim 1 \mu \mathrm{g}$ for use with $10 \mu \mathrm{L}(20 \mu \mathrm{L}$ of $50 \%$ slurry) of Protein A or G resin and $0.5-5 \mathrm{mg}$ of cell lysate prepared from $1 \times$ $10^{6}$ to $5 \times 10^{6}$ cells.

When using a polyclonal antibody, the immunoprecipitation reaction volume is seldom of concern because the antibody's avidity is high enough to allow for sufficient binding and eventual removal of the antigen, even from very dilute sample solutions. Similar approaches are possible using monoclonal antibodies displaying a high affinity for the antigen; however, low-affinity monoclonal antibodies should be applied at higher concentration in a small volume to help drive the reaction. It is critically important to ensure the complete removal of cellular debris from highly concentrated cell lysates by centrifugation before the addition of the antibody and binding matrix.

Linear polypeptide sequences referred to as "epitope tags" are specifically recognized by a wide variety of polyclonal and monoclonal antibodies with high affinity. Commonly used epitope tags-including $\mathrm{His}_{6}$, FLAG, cMyc, or HA, for example-can facilitate the immunoprecipitation of proteins by incorporating the tag-specific nucleotide sequence at the amino or carboxyl terminus of the gene of interest before protein expression. Several transgenic and knock-in mice strains carrying an epitope-tagged allele of a gene have been generated, as have libraries of yeast strains carrying an epitope tag for every protein-encoding gene, illustrating the strength and usefulness of adding an epitope tag.

More than one copy or variation ( $\mathrm{His}_{6}$ versus $\mathrm{His}_{9}$ ) of the epitope tag can be added to increase the antibody's avidity by creating multiple binding sites, making it especially valuable for the use of monoclonal antibodies that recognize only a single epitope (Yang et al. 2013). The use of epitope tags displays another advantage in that the addition of excess peptide identical to the epitope tag can be applied to elute the immunoprecipitated tagged protein as well as any associated proteins from the antibody. Different epitope tags have been combined to generate tandem affinity purification (TAP) tags, permitting antigen immunoprecipitation using one antibody followed by elution of the immune complex with the corresponding peptide, before reimmunoprecipitation using an antibody specific to the second epitope tag (see Protocol: Tandem Immunoaffinity Purification Using Anti-FLAG and Anti-HA Antibodies [DeCaprio and Kohl 2019d]).

A multitude of antibodies, including mouse or rat monoclonal antibodies and rabbit, goat, or donkey polyclonal antibodies, against many commonly used epitope tags are commercially available. It should be noted that not all antibodies generated against a specific epitope tag are equivalent, with potential antibody performance differences relating to affinity and nonspecific associations existing between monoclonal and polyclonal antibodies as well as between two monoclonal antibodies raised against the same epitope tag. Several different sources should be surveyed when choosing an epitope tag-specific antibody to identify the best one for the particular target and application.

In addition to epitope tags, a variety of polypeptides are particularly useful as affinity tags because they bind directly to an affinity matrix without the use of an antibody. For example, maltose-binding protein (MBP) (Kato et al. 2013) and glutathione-S-transferase (GST) (Rana et al. 2013) fusion proteins bind readily to amylose and glutathione Sepharose, respectively. Even though MBP and GST add $\sim 42.5$ and $26 \mathrm{kDa}$ to the fusion protein, respectively, their use is widely applied, promoting solubility and excellent recovery of a fusion protein. Affinity tags are especially useful when large amounts of protein for use as immunogen or screening reagent are required and can be used in combination with epitope tags to generate proteins that can be highly purified. 
Most immunoprecipitations incorporate a solid-phase immunoadsorbant matrix such as Protein A or $\mathrm{G}$ agarose to immobilize the immune complexes. Unless multimeric antigens are precipitated, only one antibody bound to the antigen will simultaneously be linked to the immunoadsorbant, reemphasizing the importance of the antibody's affinity not only for the antigen, but also for the resin. Use of the correct affinity matrix for an immunoprecipitation can significantly influence the quality thereof.

Protein A is a $56-\mathrm{kDa}$ surface protein originally identified as part of the Staphylococcus aureus cell wall (Graille et al. 2000). The relative affinity of the antibody's Fc portion to Protein A varies greatly between species, and not all subclasses of immunoglobulin $G(\operatorname{IgG})$ molecules bind to Protein A with equal affinity. Table 2 lists the relative binding affinity of different IgGs to Proteins A, G, and L.

Rabbit IgG antibodies bind extremely well to Protein A, but only rat monoclonal antibodies from the IgG2c subclass show little binding to the same. The most stable antibody-Protein A interaction at neutral $\mathrm{pH}$ is achieved by mouse IgG2a monoclonal antibodies, whereas IgG2b and IgG3 molecules show reduced binding. Although these two subclasses display a high enough affinity to allow for easy antigen detection, they do not bind tightly enough to Protein A to ensure the quantitative removal of all antibodies from solution. Mouse-derived antibodies displaying the lowest affinity for Protein A are of the IgG1 subclass, resulting in the inability to purify murine immune complexes successfully using Protein A resin.

Protein G, an immunoglobulin-binding protein expressed in streptococcal bacteria, displays a higher affinity for mouse IgG1 than Protein A, and the use of Protein G or Protein A/G mixtures can significantly improve the affinity for polyclonal antibodies from certain species (Sjobring et al. 1991). Protein L, first isolated from the surface of Peptostreptococcus magnus, can bind to the kappa ( $\kappa$ ) light chain from many species and is particularly useful for binding mouse monoclonal antibodies (Bjorck 1988).

TABLE 2. Relative binding affinity of IgG for Proteins A, G, and L




The vast majority of immunoprecipitations are performed using agarose or Sepharose beads as the solid matrix; however, the use of superparamagnetic or simply magnetic beads for immunoprecipitation purposes represents a newer approach with increasing popularity as an alternative to agarose or Sepharose beads for immunoprecipitation applications. Unlike agarose or Sepharose, magnetic beads are solid and do not have a porous center, thus limiting the surface area for antibody attachment. However, depending on the type of magnetic bead, their size of $1-4 \mu \mathrm{m}$ is significantly smaller than that of agarose or Sepharose beads, and the greater number of magnetic beads per volume than agarose or Sepharose beads collectively gives magnetic beads an effective surface area-to-volume ratio for optimum antibody binding.

Magnetic beads are separated based on size uniformity and are classified into monodisperse and polydisperse beads. Monodisperse beads, commonly referred to as microbeads, are uniform in size and shape and therefore show identical physical characteristics, including antibody-binding capacity and level of attraction to magnets. Although similar in size to monodisperse beads, polydisperse beads vary in size ranging from 1 to $4 \mu \mathrm{m}$, a factor that can influence their antibody-binding capacity and magnetic capture. Both types of beads are commercially available for immunoprecipitation applications; however, the higher-quality monodisperse magnetic beads are more suitable for automated applications because of their consistent size, shape, and performance.

The cross-linking of Proteins A, G, A/G, or L onto magnetic beads (Dynabeads; Life Technologies) represents a valuable alternative to the use of agarose or Sepharose beads, because proteins can be immunoprecipitated from smaller cell lysate volumes in a relative short period of time. These nonporous beads are less likely to adsorb nonspecific proteins and can significantly contribute to increasing the yield of labile protein complexes. Although clear benefits of using magnetic beads include an increased reaction speed, the gentler sample handling exclusive of centrifugation and the potential for automation make this technology a very attractive alternative for use in immunoprecipitations (see the Discussion section in Protocol: Immunoprecipitation [DeCaprio and Kohl 2017d]).

To overcome the low affinity of an antibody for the matrix, two different strategies are generally applied: (1) the use of intermediate antibodies and (2) the use of Protein G, a combination of Protein A/G, or Protein L. An antibody's low affinity to Protein A can be circumvented by the addition of intermediate antibodies, such as rabbit anti-mouse or rabbit anti-rat immunoglobulin antibodies. Rabbit-derived antibodies display a very high affinity for Protein A, and if incorporated into the immunoprecipitation, they provide an enhanced multivalent bridging interaction between the antigen-specific antibody and the matrix. However, caution is advised regarding the incorporation of nonimmunoaffinity-purified, anti-immunoglobulin polyclonal antibody, including a potential increase in background. Anti-immunoglobulin antibody titration for the determination of the minimum amount needed to bind all primary antibody is highly recommended.

No supplementation of polyclonal antibodies derived from humans, rabbits, guinea pigs, or pigs is required when used in combination with Protein A, and polyclonal antibodies generated in horses, cows, mice, hamsters, rats, or sheep only require the occasional addition of anti-immunoglobulin antibodies. Chicken- or goat-derived polyclonal antibodies should always be supplemented. Targetspecific monoclonal antibodies including all rat subclasses as well as all mouse subclasses other than IgG2a should always be supplemented for the quantitative precipitation of the antibody. Please consult Table 2 on the relative binding affinity of different IgGs to Proteins A, G, and L to determine whether rabbit anti-immunoglobulin antibodies should be added to the antigen-specific primary immunoglobulin to improve binding to Protein A.

Polyclonal and monoclonal antibodies can be directly cross-linked to agarose or Sepharose beads, thus permitting elution of the immunoprecipitated antigen without releasing the immunoglobulin into the eluate. This significantly reduces the contribution of immunoglobulin heavy and light chains in the final analyses and is particularly useful when performing mass spectroscopy or western blotting of the immunoprecipitation. Minimal distortion will be observed upon protein separation by SDSPAGE, and the potential detection of either heavy or light chains by the secondary detection antibody in the western blot is significantly reduced. Notably, antibodies cross-linked to beads can be reused if the elution conditions are sufficiently mild, thereby conserving valuable or irreplaceable reagents. 
The intermediate goal of any immunoprecipitation is to purify the target protein from a complex mixture, followed by the visualization and identification of the target and/or its associated factors and determination of its potential activity. The identification of immunoprecipitated proteins can be achieved by several different methods, with the resolution of the protein by SDS-polyacrylamide gel electrophoresis being the most common. Precast acrylamide gels are commercially available and are differentiated in the percentage of polyacrylamide present in the resolving gel, with a lower percentage of $4 \%, 6 \%, 8 \%$, or $10 \%$ typically being used for the separation of larger proteins. The resolution of smaller proteins requires a higher polyacrylamide percentage available in the form of $12 \%, 15 \%$, and $20 \%$ gels. Should no information regarding the protein's molecular weight be available, the antigen should be resolved using gradient polyacrylamide gels ranging from $4 \%$ to $20 \%, 8 \%$ to $16 \%$, or $10 \%$ to $20 \%$ according to the manufacturer's instruction.

After electrophoresis, resolved immunoprecipitated proteins can be visualized by staining the gel with Coomassie Blue or silver stain. Alternatively, resolved proteins can be transferred onto a nitrocellulose or PVDF membrane for western blot analysis using primary and/or secondary antibodies or through visualization by autoradiography or other radiosensitive imagers of immunoprecipitated radiolabeled proteins. Additionally, identification of immunoprecipitated proteins using mass spectroscopy can be achieved by direct analysis of the immunoprecipitation or from stained protein bands excised from SDS-polyacrylamide gels.

It is also possible to further manipulate immunoprecipitated proteins before any analysis. Enzyme-mediated reactions can be performed directly on the immunoprecipitated material bound to the solid-phase matrix, facilitated through buffer exchanges and the addition of enzymes, cations, adenosine triphosphate (ATP), and other cofactors, while simultaneously removing excess proteins upon completion of the reaction. On occasion, the antigen-specific antibody and/or the resin beads can interfere with the reaction; however, for the most part, this strategy can be extremely useful.

Any soluble polypeptide found within a cell can be purified to a sufficient degree by performance of an immunoprecipitation, resulting in a specific band(s) on an SDS-polyacrylamide gel. The quality of an immunoprecipitation can, however, be affected by several variables that can be optimized by the researcher, including (1) the antigen concentration in the cell and the lysate, (2) the presence and abundance of particulate debris and interacting proteins, (3) the antibody's affinity for the target, and (4) the affinity matrix used for the immunoprecipitation (see Table 2).

It is of critical importance to optimize the binding of the antibody to its specific target during an immunoprecipitation, a requirement that is often greatly influenced by the factors listed above. Abundant proteins are easier to detect and analyze by immunoprecipitation than rare proteins. Rare cellular proteins under investigation often necessitate that associated immunoprecipitations achieve purifications on the order of 100,000- to 1,000,000-fold. Although this level of purification would be difficult to achieve using other techniques, by coupling immunoprecipitation and SDSPAGE techniques, such rare polypeptide chains can be identified.

The next most important factor potentially influencing the quality of an immunoprecipitation involves the nonspecific interactions established by insoluble debris present in the cell lysate. Insoluble cellular debris, such as chromatin and cytoskeletal material, can be removed by repeated centrifugation at $15,000 \mathrm{~g}$ for $10 \mathrm{~min}$ if required. Similarly, centrifugation of the antibody preparation can ensure removal of precipitated antibody and/or insoluble material before use. The cell lysate and/or antibody supernatant should be recovered carefully without disruption of the formed pellet and the reagents transferred into clean tubes before adding these to the binding matrix. In addition, Mycoplasma contamination present in mammalian cell cultures can significantly increase the background signal 
in an immunoprecipitation, and thus cell cultures are required to be free of Mycoplasma before the preparation of cell lysates.

Furthermore, the quality of an immunoprecipitation is greatly influenced by the presence and abundance of nonspecific interacting proteins, such as actin, tubulin, vinculin, and myosin. These proteins can assemble into polymers and other complex structures, which could potentially form lowaffinity interactions with the antigen, the antibody, the affinity matrix, or even the tube in which the immunoprecipitation is performed and are difficult to be completely removed during the washing steps. (For example, polypropylene tubes have a reduced tendency to bind nonspecific proteins, and their use for an immunoprecipitation is preferred over that of polystyrene tubes.) Care must be exerted to minimize the presence of these low-affinity but highly abundant proteins, which can significantly contribute to the background signal of an immunoprecipitation. Given these limitations, insoluble or highly polymerized antigens represent a challenge in the investigation by immunoprecipitation, and extreme care must be applied when studying these proteins to forego inaccurate conclusions resulting from nonspecific protein interactions.

The third factor affecting the purification of an antigen is the antibody's affinity for the antigen. Unlike other assays in which the antigen is present at high concentrations, immunoprecipitations rely on the formation of the antigen-antibody complex in solution at relatively low concentrations of the antigen. In practice, this translates into the use of antibodies displaying an affinity of $10^{8} \mathrm{~mol}^{-1}$ or higher for the successful performance of a quantitative immunoprecipitation. Antibodies displaying a relative affinity of $10^{7} \mathrm{~mol}^{-1}$ for the antigen are capable of detecting the target; however, they will not be able to facilitate quantitative removal of the antigen from solution. Antibodies with affinities of $10^{6}$ $\mathrm{mol}^{-1}$ or lower might be functional for other assays, but they are not suitable for the performance of an immunoprecipitation. Thus, care should be taken upon selection of a suitable antibody to be incorporated into this assay.

\section{BACKGROUND PROBLEMS}

The most frequent concerns regarding immunoprecipitations address methods for lowering the number and type of background proteins contaminating washed immune complexes. Background problems arise from many different sources and can be classified as specific or nonspecific.

Specific Background

It is important to remember that polyclonal antibody preparations could contain spurious antibodies capable of detecting antigens, resulting in specific background bands. Also, a polyclonal antibody preparation may display cross-reactivity to unrelated antigens present in the cell lysate. Affinitypurified polyclonal antibodies or monoclonal antibodies generated against a short peptide sequence may specifically recognize the full-length target protein; however, the potential capability of the antibodies to detect an unrelated protein containing an identical or highly similar epitope spanning four or five amino acids cannot be excluded. In some cases, common epitopes may form part of highly homologous domains between antigens. Given the frequency of these cross-reactions, the precipitation of an unexpected polypeptide should be treated as a contaminant until proven otherwise.

\section{Nonspecific Background}

Several sources contribute to the presence of nonspecific background proteins, including weak interactions of highly abundant proteins with the immune complexes or the matrix. Nonspecific particulate matter contained in the cell lysate can trap the antigen of interest and subsequently stick to the formed immune complex, whereas aggregated or polymerized proteins contained within the cell lysate can become trapped within the latticework of the antigen-antibody-matrix complex. Although these larger contaminants can be removed by centrifugation of the cell lysate and/or immune complexes, they can remain bound to the plasticware used during the centrifugation process. 
A control showing that the presence of the protein target in the immunoprecipitation is strictly dependent on the incorporated specific antibody and not a result of nonspecific associations with immunoglobulin or the matrix is essential for every immunoprecipitation experiment. The correct controls for immunoprecipitation reactions consist of antibody preparations closely resembling the specific antibody; for example, polyclonal serum should be compared with other polyclonal serum batches of the same species. A prebleed serum preparation obtained before the first immunization from the same animal used for the antibody development is the optimal control. Controls for the use of monoclonal antibodies require that antibody preparations be taken from the same source as the specific antibody (i.e., hybridoma tissue culture supernatant versus hybridoma tissue culture supernatant, ascites versus ascites, or purified immunoglobulin versus purified immunoglobulin) and should be of the same isotype as the specific antibody (i.e., IgG1 versus IgG1). Exclusion of an antibody, as is customary in the enzyme immunoassay (EIA), in which the antigen is incubated in the absence of primary antibody, is not sufficient as an adequate control for comparing immunoprecipitation efficiencies. Tissue culture supernatant derived from the parental myeloma does not serve as an appropriate control because it contains no antibodies; however, a variety of suitable control hybridoma cell lines are available from the American Type Culture Collection (ATCC).

An extremely valuable control for any immunoprecipitation is incorporating an aliquot of nonimmunoprecipitated cell lysate alongside the immunoprecipitated protein in the SDS-PAGE and western blot, providing useful information about the relative abundance of the antigen as well as the efficiency of the immunoprecipitation, and it may reveal if the immunoprecipitation enriched for a subset of the total target protein.

\section{REFERENCES}

Barrett B, Wood PA, Volwiler W. 1960. Quantitation of $\gamma$ globulins in human serum by immunoprecipitation. J Lab Clin Med 55: 605-615.

Bjorck L. 1988. Protein L. A novel bacterial cell wall protein with affinity for Ig L chains. J Immunol 140: 1194-1197.

DeCaprio J, Kohl TO. 2017a. Metabolic labeling of protein antigens with $\left[{ }^{35}\right.$ S]methionine. Cold Spring Harb Protoc doi: 10.1101/pdb .prot098517.

DeCaprio J, Kohl TO. 2017b. Detergent lysis of tissue culture cells for immunoprecipitation. Cold Spring Harb Protoc doi: 10.1101/pdb .prot098558.

DeCaprio J, Kohl TO. 2017c. Detergent lysis of animal tissues for immunoprecipitation. Cold Spring Harb Protoc doi: 10.1101/pdb.prot098566.

DeCaprio J, Kohl TO. 2017d. Immunoprecipitation. Cold Spring Harb Protoc doi: 10.1101/pdb.prot098640.

DeCaprio J, Kohl TO. 2018a. Metabolic labeling of protein antigens with $\left[{ }^{32} \mathrm{P}\right]$ orthophosphate. Cold Spring Harb Protoc doi: 10.1101/pdb .prot098533.

DeCaprio J, Kohl TO. 2018b. Pulse-chase labeling of protein antigens with ${ }^{[35}$ S $]$ methionine. Cold Spring Harb Protoc doi: 10.1101/pdb .prot098525.

DeCaprio J, Kohl TO. 2019a. Using dounce homogenization to lyse cells for immunoprecipitation. Cold Spring Harb Protoc doi: 10.1101/pdb .prot098574.

DeCaprio J, Kohl TO. 2019b. Cross-linking antibodies to beads using dimethyl pimelimidate (DMP). Cold Spring Harb Protoc doi: 10.1101/ pdb.prot098624.

DeCaprio J, Kohl TO. 2019c. Cross-linking antibodies to beads with disuccinimidyl suberate (DSS). Cold Spring Harb Protoc doi: 10.1101/pdb .prot098632.

DeCaprio J, Kohl TO. 2019d. Tandem immunoaffinity purification using anti-FLAG and anti-HA antibodies. Cold Spring Harb Protoc doi: 10.1101/pdb.prot098657.

DeCaprio J, Kohl TO. 2020a. Chromatin immunoprecipitation. Cold Spring Harb Protoc doi: 10.1101/pdb.prot098665.
DeCaprio J, Kohl TO. 2020b. Differential detergent lysis of cellular fractions for immunoprecipitation. Cold Spring Harb Protoc doi: 10.1101/pdb .prot098582.

DeCaprio J, Kohl TO. 2020c. Denaturing lysis of cells for immunoprecipitation. Cold Spring Harb Protoc doi: 10.1101/pdb.prot098616.

DeCaprio J, Kohl TO. 2020d. Lysing yeast cells with glass beads for immunoprecipitation. Cold Spring Harb Protoc doi: 10.1101/pdb prot098590.

DeCaprio J, Kohl TO. 2020e. Lysing yeast cells for immunoprecipitation using a coffee grinder. Cold Spring Harb Protoc doi: 10.1101/pdb .prot98608.

Graille M, Stura EA, Corper AL, Sutton BJ, Taussig MJ, Charbonnier J-B, Silverman GJ. 2000. Crystal structure of a Staphylococcus aureus protein A domain complexed with the Fab fragment of a human IgM antibody: Structural basis for recognition of B-cell receptors and superantigen activity. Proc Natl Acad Sci 97: 5399-5404.

Kato M, Ishibashi K, Kobayashi C, Ishikawa M, Katoh E. 2013. Expression, purification, and functional characterization of an $\mathrm{N}$-terminal fragment of the tomato mosaic virus resistance protein Tm-1. Prot Expr Purif 89: $1-6$.

Litovchick L. 2019. Freezing cell pellets for large-scale immunoprecipitation. Cold Spring Harb Protoc doi: 10.1101/pdb.prot098541.

Litovchick L. 2020. Immunoblotting. Cold Spring Harb Protoc doi: 10.1101/ pdb.top098392.

Rana S, Lattoo SK, Dhar N, Razdan S, Bhat WW, Dhar RS, Vishwakarma R. 2013. NADPH-cytochrome P450 reductase: Molecular cloning and functional characterization of two paralogs from Withania somnifera (L.) Dunal. PLoS One 8: e57068.

Sjobring U, Bjorck L, Kastern W. 1991. Streptococcal protein G. Gene structure and protein binding properties. J Biol Chem 266: 399-405.

Yang L, Zhou L, Zong X, Cao X, Ji X, Gu W, Zhang S. 2013. Characterization of the molecular structure, expression and bioactivity of the TNFSF13B (BAFF) gene of the South African clawed frog, Xenopus laevis. Int Immunopharmacol 15: 478-487. 


\section{Immunoprecipitation}

James DeCaprio and Thomas O. Kohl

Cold Spring Harb Protoc; doi: 10.1101/pdb.top098509

\begin{tabular}{|c|c|}
\hline $\begin{array}{r}\text { Email Alerting } \\
\text { Service }\end{array}$ & Receive free email alerts when new articles cite this article - click here. \\
\hline $\begin{array}{r}\text { Subject } \\
\text { Categories }\end{array}$ & $\begin{array}{l}\text { Browse articles on similar topics from Cold Spring Harbor Protocols. } \\
\text { Antibodies (119 articles) } \\
\text { Antibodies, general (289 articles) } \\
\text { Cell Biology, general (1382 articles) } \\
\text { Immunoprecipitation (75 articles) } \\
\text { Protein Identification and Analysis (202 articles) } \\
\text { Proteins and Proteomics, general (575 articles) }\end{array}$ \\
\hline
\end{tabular}

